PROCEEDINGS OF THE

AMERICAN MATHEMATICAL SOCIETY

Volume 133, Number 9, Pages 2779-2782

S 0002-9939(05)07820-2

Article electronically published on March 22, 2005

\title{
WECKEN PROPERTY FOR ROOTS
}

\author{
DACIBERG GONÇALVES AND PETER WONG
}

(Communicated by Paul Goerss)

\begin{abstract}
We show that maps from a compact space into a topological manifold which have geometric Nielsen root number zero satisfy the Wecken property, i.e., $N(f ; a)=0 \Rightarrow f \sim g$ such that $g^{-1}(a)=\emptyset$.
\end{abstract}

\section{INTRODUCTION}

Let $f: M \rightarrow N$ be a map between two closed smooth oriented $n$-manifolds and $a \in N$ be a regular value. It is well known that the topological Brouwer degree $\operatorname{deg} f$ is an algebraic count of the set $f^{-1}(a)$ of preimages. In 1930, H. Hopf [8] generalized this degree for maps between manifolds that are not necessarily orientable. He introduced his theory, now known as Hopf degree theory, using Nielsen root theory. Following the work of J. Nielsen on fixed point theory, Hopf introduced an equivalence relation on the set $f^{-1}(a)$ and defined a Nielsen root number, denoted by $N(f ; a)$. This number is a homotopy invariant and is a lower bound for the cardinality of $f^{-1}(a)$ among all maps homotopic to $f$. In particular, Hopf showed that if the degree $\operatorname{deg} f$ is zero, then $N(f ; a)=0$.

For fixed points, F. Wecken in the early 1940's showed that for self-maps of a compact triangulated manifold of dimension at least three, the Nielsen number, which is a lower bound for the number of fixed points, can be realized in the homotopy class of the given map, i.e., there exists a map homotopic to the given map with exactly the Nielsen number as the number of fixed points. In particular, the vanishing of the Nielsen (fixed point) number is equivalent to showing that the given map is deformable to be fixed point free. Subsequently in 1955, H. Schirmer [9] proved a Wecken type theorem for coincidences of two maps between triangulated closed orientable manifolds of the same dimension. When the target space is a manifold, the Nielsen root theory is indeed a special case of the Nielsen coincidence theory where one of the maps is a constant map. Thus, if $f: M \rightarrow N$ is a map between triangulated closed orientable $n$-manifolds, then $N(f ; a)=0 \Rightarrow \exists g \sim f$ such that $g^{-1}(a)=\emptyset$.

Received by the editors October 7, 2003 and, in revised form, April 28, 2004.

2000 Mathematics Subject Classification. Primary 55M20, 55R20, 55T10; Secondary 55S35.

Key words and phrases. Nielsen number, Reidemeister number, Wecken property.

This work was conducted during the first author's visit to Bates College, April 11-23, 2003, and the second author's visits to São Paulo, May 13-20, 2003 and April 27 - May 4, 2004. The first author's visit was partially supported by the "Projeto temático Topologia Algébrica e GeométricaFAPESP". The second author's visits were partially supported by a grant from Bates College, the N.S.F., and the "Projeto temático Topologia Algébrica e Geométrica-FAPESP". 
In the late 1960 's, R. Brooks independently discovered Hopf's result by generalizing Nielsen root theory (see [1], 2]). Now let $f: X \rightarrow Y$ be a map between two topological spaces and let $a \in Y$. Two roots $x, y \in f^{-1}(a)$ are said to be equivalent if there exists a path $C:[0,1] \rightarrow X$ such that $C(0)=x, C(1)=y$ and $f \circ C$ is homotopic to the constant path at $a$, relative to the endpoints. These equivalence classes are called root classes. In order to extend the Nielsen theory to more general spaces, Brooks used the following index-free notion of essentiality: a root class $\mathcal{F}$ is essential if for any given homotopy $f \sim_{H} f_{1}$, there exist a root $y$ of $f_{1}$ and a path $C$ in $X \times[0,1]$ with $C(0) \in \mathcal{F}, C(1)=y$ such that $H \circ C$ is homotopic to the constant loop at $a$, i.e., $\mathcal{F}$ is related to some roots of $f_{1}$. In other words, a root class $\mathcal{F}$ is inessential if it disappears under some homotopy of $f$. The (geometric) Nielsen root number is defined as the number of essential root classes. Since it reduces to the classical one when the spaces are oriented manifolds of the same dimension, we use the same notation $N(f ; a)$ to denote this geometric Nielsen root number.

While Nielsen root theory has been given much attention in recent years (see e.g. 4]), the Wecken property for roots has not been fully investigated in situations where the spaces are not manifolds or are manifolds of different dimensions. R. Brooks gave a partial result in 2 in which he showed that every map $f$ from an $n$ complex to an $n$-manifold is homotopic to a map $g$ with exactly $N(f ; a)$ root classes. In this note, we improve this result for the case when $N(f ; a)=0$. In fact, we show that if the target space is a topological manifold, then $N(f ; a)=0 \Rightarrow \exists g \sim f$ such that $g^{-1}(a)=\emptyset$ for every $f: X \rightarrow Y$.

\section{Main Results}

We now show that if the target space is a topological manifold, then the vanishing of the Nielsen root number is sufficient to guarantee the existence of a root free map in the homotopy class of the given map. By a topological manifold, we mean a separable metric space that is locally euclidean.

Theorem 2.1. Let $f: X \rightarrow Y$ be a map between two path connected spaces. Suppose $X$ is compact, $Y$ is a topological n-manifold (not necessarily compact), and $a \in Y$ is a point in $Y$. If the Nielsen root number $N(f ; a)=0$, then there exists a map $g \sim f$ such that $g^{-1}(a)=\emptyset$.

Proof. Suppose $f^{-1}(a)$ is nonempty; otherwise there is nothing to prove. Let $p$ : $\hat{Y} \rightarrow Y$ be the cover corresponding to the subgroup $f_{\sharp}\left(\pi_{1}(X)\right)$ in $\pi_{1}(Y)$. Since $N(f ; a)=0$, the root classes are inessential. (In fact, Brooks [1] showed that $N(f ; a) \neq 0$ implies that all root classes are essential.) Consider an inessential root class $\gamma$ of $f$. Then $f$ is homotopic to a map $f^{\prime}$ such that $\gamma$ is not related to any roots of $f^{\prime}$. Let $\hat{a} \in p^{-1}(a)$. It follows that there exists a lift $\hat{f}^{\prime}: X \rightarrow \hat{Y}$ such that $p \circ \hat{f}^{\prime}=f^{\prime}$ and $\hat{a} \notin \hat{f}^{\prime}(X)$.

By a result of Doyle and Hocking [5], since $\hat{Y}$ is a manifold, $\hat{Y}=P \cup C$, a disjoint union of $P$ and $C$ where $P$ is homeomorphic to the Euclidean $n$-space (open $n$-cell) and $C$ is a nowhere dense closed subset so that $\operatorname{dim} C \leq n-1$. Since $C$ is nowhere dense and $p$ is a local homeomorphism, without loss of generality, we may choose the point $a$ so that $p^{-1}(a) \subset P$. Since $\hat{f}^{\prime}(X)$ is compact, there are only a finite number of points $\hat{a}_{1}, \hat{a}_{2}, \ldots, \hat{a}_{k} \in p^{-1}(a)$ that lie inside $\hat{f}^{\prime}(X) \cap P$. Now, we can use the fact that $P$ is a manifold and get a closed $n$-disk $D^{n} \subset P$ containing $\hat{a}$ and all of the $\hat{a}_{i}$ (similar to Lemma 1 of [5], p. 469). Then, we use $\hat{a}$ as the center of the 
disk and get a strong deformation retract of $D^{n}-\{\hat{a}\}$ to its boundary $\partial D^{n}$. Since $\hat{a} \notin \hat{f}^{\prime}(X)$, we can compose $\hat{f}^{\prime}$ with the retraction and obtain a map $\varphi$ homotopic to $\hat{f}^{\prime}$ such that $p^{-1}(a) \cap \varphi(X)=\emptyset$. Now let $g=p \circ \varphi$ so that $f \sim g$ and $g^{-1}(a)=\emptyset$.

Corollary 2.2. If the subgroup $f_{\sharp}\left(\pi_{1}(X)\right)$ has infinite index in $\pi_{1}(Y)$, then $f$ is homotopic to a map $g$ such that $g^{-1}(a)=\emptyset$.

Proof. Since $Y$ is a manifold, it follows from [1] that $N(f ; a)>0 \Rightarrow R(f ; a)=$ $N(f ; a)<\infty$ where $R(f ; a)=\left[\pi_{1}(Y): f_{\sharp}\left(\pi_{1}(X)\right)\right]$ is the Reidemeister number. Now $R(f ; a)=\infty$; therefore $N(f ; a)=0$ and the result follows from Theorem 2.1.

With additional hypotheses, Theorem 2.1 can be strengthened as follows.

Theorem 2.3. Let $f: X \rightarrow Y, a \in Y$ be as in Theorem 2.1 with $N(f ; a)=0$. If $Y$ is closed and $R(f ; a)<\infty$ or if $Y$ is triangulable, then $f$ is homotopic to a root free map $g$ such that $\operatorname{dim} g(X) \leq n-1$.

Proof. Case I: $Y$ is closed and $R(f ; b)<\infty$.

A theorem of Brown and Casler 3] asserts that every closed topological $n$ manifold $M^{n}$ is the image of the closed $n$-cell such that $M^{n}$ can be decomposed into a disjoint union of an open $n$-cell and a closed subset of dimension at most $n-1$. (This result is a refinement of the result of Doyle and Hocking [5] in the case of closed topological manifolds.) More precisely, there exists a map $\varphi: I^{n} \rightarrow M^{n}$ such that $M^{n}=\varphi\left(I^{n}\right)$, (i) $\varphi \mid$ int $I^{n}$ is a homeomorphism; (ii) $\varphi^{-1}\left(\varphi\left(\partial I^{n}\right)\right)=\partial I^{n}$; (iii) $\operatorname{dim} \varphi\left(\partial I^{n}\right) \leq n-1$.

Let $\tilde{Y} \stackrel{p}{\rightarrow} Y$ be the covering which corresponds to the subgroup $f_{\#}\left(\pi_{1}(X)\right)$. Let $f_{1}$ be a map homotopic to $f$ such that one of the root classes is empty. Call $\tilde{f}_{1}$ a lifting of $f_{1}$. This means that there exists $\hat{a} \in p^{-1}(a)$ such that $\hat{a} \notin \tilde{f}_{1}(X)$. Since $R(f ; a)<\infty, \tilde{Y}$ is a closed $n$-manifold. So following [3], there exists a map $\varphi: I^{n} \rightarrow \tilde{Y}$ such that $\varphi\left(I^{n}\right)=\tilde{Y}$ and it satisfies conditions (i) - (iii). We may assume that $\hat{a} \in \varphi\left(\right.$ int $\left.I^{n}\right)$. Let $\psi: I^{n}-\varphi^{-1}(\hat{a}) \rightarrow \partial I^{n}$ be a strong deformation retraction.

Now the map

$$
\eta(x)= \begin{cases}\varphi \circ \psi \circ \varphi^{-1}, & \text { if } x \in \varphi\left(\text { int } I^{n}\right) \\ x, & \text { if } x \in \varphi\left(\partial I^{n}\right)\end{cases}
$$

is a strong deformation retraction of $\tilde{Y}-\{\hat{a}\} \rightarrow \varphi\left(\partial I^{n}\right)$. Since $\varphi\left(\partial I^{n}\right)$ is at most $(n-1)$-dimensional, the map $\tilde{f}_{1}$ is homotopic to a map $\tilde{g}$ such that $\tilde{g}(X) \cap p^{-1}(a)=\emptyset$. It follows that $f_{1}$ and hence $f$ is homotopic to a map $g$ such that $a \notin g(K)$.

Case II: $Y$ is triangulable.

Consider the map $f_{1}$ and its lift $\tilde{f}_{1}$ as in Case I. Consider a triangulation of $\tilde{Y}$ such that the projection is a simplicial map. Since $f_{1}(X)$ is compact, it is contained in a finite simplicial subcomplex. In fact, a compact triangulable $n$-manifold with boundary admits a retraction to a subcomplex which is contained in the $(n-1)$ skeleton. Together with this retraction, it is straightforward to construct a map $g \sim f$ such that $g(X)$ lies in the $(n-1)$-skeleton of $Y$ and $g^{-1}(a)=\emptyset$.

Remark 2.1. We should point out that when $N(f ; a)>0$ the number of roots within each root class may be minimized but not simultaneously (see [6]). Furthermore, 
the Wecken property for coincidences does not hold true in general, even in the case when $N(f, g)=0$ as we illustrate in the next example.

Example 2.4. Let $f: S^{11} \rightarrow S^{6}$ be a map given by the Whitehead product. It was shown in [7] that the map $f$ and itself are homotopic to maps $f_{1}, f_{2}$ that are coincidence free. However, such maps must have the property that there exists a point $x \in S^{11}$ such that $f_{1}(x)=-f_{2}(x)$. Thus, if $p$ denotes the covering map from $S^{6}$ to $\mathbb{R} P^{6}$, then $p \circ f_{1}$ and $p \circ f_{2}$ must have a coincidence. In other words, $p \circ f$ and $p \circ f$ cannot be made coincidence free. On the other hand, there are two coincidence classes, one of which is empty and the other is all of $S^{11}$, and they correspond to $\pi_{1}\left(\mathbb{R} P^{6}\right)$. These two classes are related by homotopy in the sense of [2]. This fact can be seen by the fact that the two lifts, namely, $f$ and $A \circ f$, of $f$ are homotopic where $A$ is the antipodal map on $S^{6}$. Thus, $N(p \circ f, p \circ f)=0$.

\section{REFERENCES}

[1] R. Brooks, Certain subgroups of the fundamental group and the number of roots of $f(x)=a$, Amer. J. Math. 95 (1973), 720-728. MR0346777 (49:11501)

[2] (1973), 101-108. MR0331373 (48:9706)

[3] M. Brown, A mapping theorem for untriangulated manifolds, in Topology of 3-manifolds pp. 92-94, M.K. Fort, Jr. (editor), Prentice-Hall, Englewood Cliffs, New Jersey, 1962. MR0158374 $(28: 1599)$

[4] R. F. Brown and H. Schirmer, Nielsen root theory and Hopf degree theory, Pacific J. Math. 198 (2001), 49-80. MR1831972 (2002c:55005)

[5] P. Doyle and J. Hocking, A decomposition theorem for $n$-dimensional manifolds, Proc. Amer. Math. Soc. 13 (1962), 469-471. MR0141101 (25:4514)

[6] D. Gonçalves and C. Aniz, The minimizing of the Nielsen root classes, Central European J. Math. 2 (2004), 112-122. MR2041673 (2004k:55004)

[7] D. Gonçalves and D. Randall, Self-coincidence of maps from $S^{q}$-bundles over $S^{n}$ to $S^{n}$, Boletin de la Sociedad Matematica Mexicana, to appear.

[8] H. Hopf, Zur Topologie der Abbildungen von Mannigfaltigkeiten, Zweiter Teil, Math. Ann., 102 (1930), 562-623.

[9] H. Schirmer, Mindestzahlen von Koinzidenzpunkten, J. Reine Angew. Math. 194 (1955), 21-39. MR0073172 (17:394e)

Department de Matemática - ime - UsP, Caixa Postal 66.281, CeP 05311-970, São PAULO - SP, BRASIL

E-mail address: dlgoncal@ime.usp.br

Department of Mathematics, Bates College, Lewiston, Maine 04240

E-mail address: pwong@bates.edu 